\title{
Pulse oximetry at the roadside: a study of pulse oximetry in immediate care
}

\author{
Paul Silverston
}

\begin{abstract}
The measurement of tissue oxygen saturation with a pulse oximeter is of proved value in the hospital setting. The development of a portable oximeter has allowed this investigation to be performed during the prehospital phase of a patient's care. Pulse oximetry was performed at the roadside in 25 patients with abnormal trauma scores and found to be of benefit in detecting and monitoring hypoxia in patients with airway obstruction, depressed respiration due to head injury, and, in particular, with closed chest injuries. There were no practical difficulties associated with the use of the instrument either at the roadside or in a moving ambulance.
\end{abstract}

The portable pulse oximeter is a valuable aid in the prehospital monitoring of patients with trauma.

\section{Introduction}

The Mid Anglia General Practitioner Accident Service covers a mainly rural area based on the County of Cambridgeshire, and the 80 participating doctors attend some 2500 accidents a year.' The county is crossed by trunk roads carrying a large volume of traffic, and many of the accidents occur at high speeds, resulting in prolonged entrapment of seriously injured casualties. In our area whenever an ambulance is mobilised to a road accident the nearest immediate care doctor is dispatched at the same time. Diagnosis and monitoring of the patient's condition at the roadside and on the way to hospital can often be difficult. A trial of pulse oximetry was therefore carried out to see whether measuring the tissue oxygen saturation $\left(\mathrm{SaO}_{2}\right)$ would be of benefit in these cases.

Correspondence to: Mill Road Farm, Little

Wilbraham, Cambridge CB1 5LG.

\section{Patients and methods}

The Biox 3700 is a portable battery powered pulse oximeter which is widely used in hospital. Its features

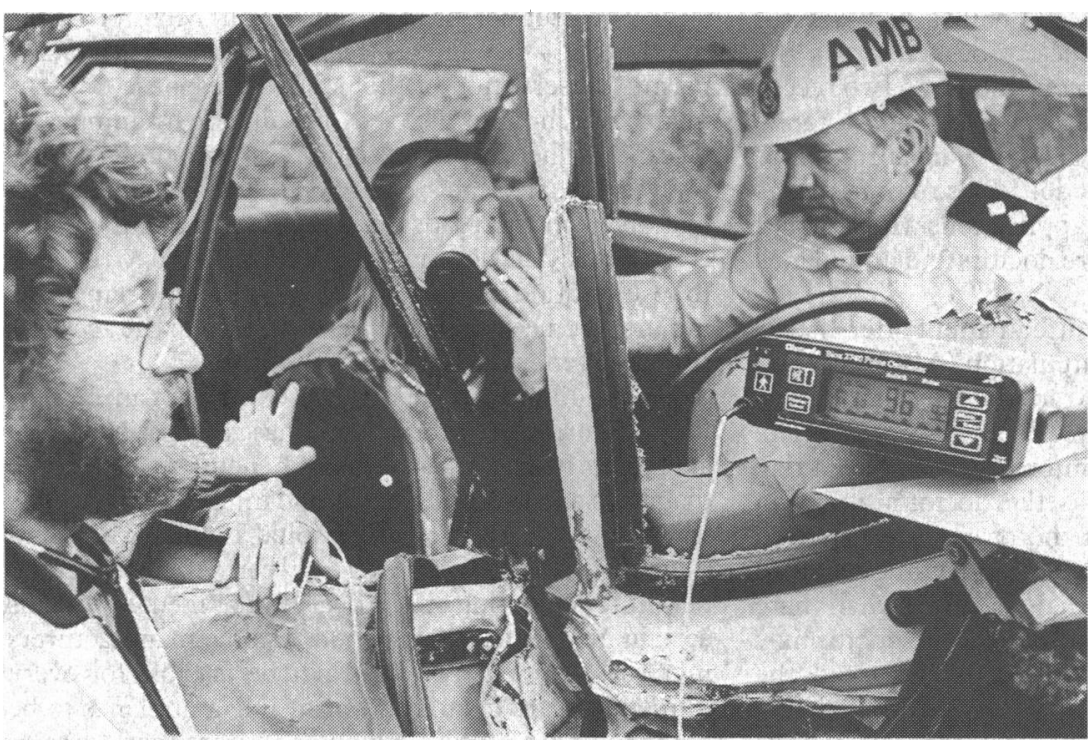

Simulated use of the Ohmeda Biox 3740 pulse oximeter include a graphic display of signal strength and plethysmographic waveform, which enable the user to make a continuous assessment of signal integrity. $\mathrm{SaO}_{2}$ and pulse rate are displayed, and a visual display of trends in $\mathrm{SaO}_{2}$ from the previous 60 or 20 minutes may be accessed, allowing review of the effectiveness of treatment. Non-invasive measurement of the $\mathrm{SaO}_{2}$ is achieved with a clip probe attached to an ear lobe or finger.

During the summer of 1988 an Ohmeda Biox 3700 pulse oximeter was carried by a member of the Mid Anglia General Practitioner Accident Service and the $\mathrm{SaO}_{2}$ measured in 25 consecutive patients with altered trauma scores. ${ }^{2}$ In 19 cases this doctor was the first medically trained person to arrive at the scene, in most cases within 10 minutes of the emergency 999 call. The ambulance arrived before the doctor in four cases and in the remaining two another immediate care doctor was already at the scene and had requested on site oximetry. Whenever possible pulse oximetry was begun before treatment.

\section{Results}

Of the 25 patients studied, four had initial $\mathrm{SaO}_{2}$ readings of less than $75 \%$, and in two cases the readings were below $65 \%$. In all four cases hypoxia was due to partial airway obstruction from either vomit or blood. In the two patients with readings below $65 \%$ airway obstruction was aggravated by hypoventilation as a result of severe head injury. In all four cases aspiration of the airway and insertion of either an oropharyngeal airway (three cases) or a pharyngeal tracheal lumen airway together with high flow oxygen resulted in a rise in the $\mathrm{SaO}_{2}{ }^{3}$ The two patients with hypoventilation secondary to severe head injury required intermittent positive pressure ventilation with oxygen to bring $\mathrm{SaO}_{2}$ back to normal, whereas the two patients with airway obstruction alone required only airway care and oxygen to achieve this. The pulse oximeter was of particular value in monitoring the two patients receiving intermittent positive pressure ventilation as both patients developed complications on the way to hospital. In the first case a fall in $\mathrm{SaO}_{2}$ was found to be due to an inadequate pharyngeal tracheal lumen airway cuff seal, which was quickly rectified. In the second case a fall in $\mathrm{SaO}_{2}$ alerted the doctor to the development of a pneumothorax, which was relieved by the insertion of a chest drain.

Seven patients had an initial $\mathrm{SaO}_{2}$ of $75-90 \%$, of whom six were trapped with multiple injuries. Intensive resuscitation was begun with airway care, oxygenation, ventilation (when appropriate), fluid replacement, analgesia and sedation, and application of the antishock suit on release (three cases). ${ }^{+}$Five of the seven patients had serious chest injuries (flail chest; pneumothorax or haemothorax, or both; severe pulmonary contusion), and in each case the $\mathrm{SaO}_{2}$ failed to rise above $91 \%$ with initial resuscitation measures. In the other two cases volume replacement and oxygenation resulted in a normal $\mathrm{SaO}_{2}$ and neither patient had serious chest injury. 
Six patients had an initial $\mathrm{SaO}_{2}$ of between $90 \%$ and $95 \%$, of whom two had concussion alone, two had concussion and fracture of a long bone, one had inspiratory chest pain from several rib fractures, and the last had multiple injuries. All six patients received oxygen by mask, and the $\mathrm{SaO}_{2}$ returned to normal in the four with concussion. The patient with hypoventilation secondary to pain from rib fractures required $7.5 \mathrm{mg}$ morphine intravenously before he was able to breathe enough oxygen to improve his $\mathrm{SaO}_{2}$. The patient with multiple injuries was initially found to have an $\mathrm{SaO}_{2}$ of $93 \%$ (breathing air), which returned to normal when he was given a high concentration of oxygen by facemask. On the way to hospital his $\mathrm{SaO}_{2}$ fell to $84 \%$, and examination showed a pneumothorax, which was managed with a chest drain.

The remaining eight patients had $\mathrm{SaO}_{2}$ readings of between $95 \%$ and $99 \%$, of whom two had concussion alone, two had concussion and fracture of a long bone, three had multiple fractures, and one was a boy whose arm was impaled on a railing.

\section{Discussion}

Pulse oximetry has been used in hospital for several years and is a reliable means of detecting hypoxia in the absence of severe hypothermia and hypotension (mean blood pressure less than $50 \mathrm{~mm} \mathrm{Hg}$ ). ${ }^{-7}$ The Biox 3700 pulse oximeter was used in a hospital study of patients with low cardiac output states and with reduced peripheral perfusion in which 18 patients having open heart surgery were monitored (H Palve, 19th congress of the Scandinavian Society of Anaesthesiologists, July 1987; H Palve, A Vuori, forum on pulse oximetry, Munich, September 1987). Accurate $\mathrm{SaO}_{2}$ readings were obtained with the Biox 3700 at a peripheral body temperature of $24 \cdot 8^{\circ} \mathrm{C}$ and a cardiac index of $2.3 \mathrm{l} / \mathrm{min} /$ $\mathrm{m}^{2}$. When a vasodilating cream was applied to the skin under the probe it was possible to obtain accurate readings at a peripheral body temperature of $22.7^{\circ} \mathrm{C}$ and a cardiac index of $2 \cdot 0 \mathrm{l} / \mathrm{min} / \mathrm{m}^{2}$. It was found that if the Biox 3700 could detect a pulse it would provide an accurate $\mathrm{SaO}_{2}$ reading whatever the signal strength displayed, and fears of erroneous readings due to poor peripheral perfusion were unfounded. Either the machine gave an accurate reading or it gave none at all. It was not known whether this would be the case for other makes of pulse oximeter.

Thus though it may not be possible to perform pulse oximetry on patients with profound peripheral shutdown, it seems unlikely that this would be a source of erroneous readings. In this study no patient was hypothermic or had severe hypotension. Contamination of the skin by blood, oil, and nail varnish did not affect the performance of the probes either at the roadside or under laboratory conditions. In two recent American studies pulse oximetry was found to be reliable during both ground and air transportation of patients as well as at the roadside. ${ }^{\mathrm{x}}{ }^{9}$ Pulse oximetry was found to have several clinical applications which would be of benefit to immediate care doctors and ambulance personnel.

Initial assessment of the injured patient after checking airway, breathing, and circulation ( $\mathrm{ABC}$ protocol) should enable the rescuer rapidly to identify the compromised airway and to treat any immediate lifethreatening problem. Monitoring the airway at risk may be difficult, however, as the doctor's attention may be diverted away from the airway by the need either to perform other resuscitation techniques or to see other critically injured casualties. Also, when there is limited access to the trapped patient the doctor may have to stand aside while other rescuers extricate the casualty. In such settings remote monitoring of the $\mathrm{SaO}_{2}$ can alert the doctor to the presence of hypoxia before it becomes clinically apparent, so allowing rapid intervention. In an incident with multiple casualties a member of the public was shown how to manage an airway in a patient connected to the pulse oximeter and was instructed to inform the doctor (who was attending to another unconscious patient with an airway problem) immediately if the hypoxia alarm sounded.

Hypoventilation may not be recognised immediately and, when diagnosed, may present the rescuer with the problem of deciding whether intermittent positive pressure ventilation is indicated in a patient who is still breathing spontaneously. Measurement of the $\mathrm{SaO}_{2}$ permits rapid identification of serious hypoxia secondary to hypoventilation and allows the rescuer to decide whether intermittent positive pressure ventilation is needed. Measurement of respiratory rate and depth may be difficult and unreliable in these patients. The effect of pain from rib fractures can also be assessed, as can the patient's response to intravenous analgesics. Patients given intravenous anaesthetic agents, opiates, and sedatives are at risk from the respiratory depressant side effects of these drugs, so it is reassuring to be able to monitor the $\mathrm{SaO}_{2}$ while these agents are being given. The boy whose arm was impaled on a railing was given ketamine and midazolam and his $\mathrm{SaO}_{2}$ monitored throughout the rescue.

Assessment and monitoring of closed chest injuries is particularly difficult during the prehospital phase of a patient's care. It is often impossible to gain access to entrapped patients in order to examine them thoroughly, and even when this is not a problem noise from rescue equipment or in a moving ambulance makes listening for breath sounds extremely difficult Pulse oximetry may be used to assess and monitor these patients. Underlying pulmonary damage requiring immediate treatment can be excluded if the patient has a normal $\mathrm{SaO}_{2}$, whereas a deteriorating $\mathrm{SaO}_{2}$ (in the absence of airway obstruction) should alert the rescuer to hypoventilation secondary to central (neurological), peripheral (pulmonary, thoracic cage), or iatrogenic (drug side effect) causes. In this study all patients whose $\mathrm{SaO}_{2}$ failed to rise above $91 \%$ with initial resuscitation had serious chest injuries. Two developing pneumothoraces were detected after a fall in the $\mathrm{SaO}_{2}$ reading.

A set of baseline observations during the prehospital phase of a patient's care is of benefit to both immediate care and hospital doctors. The immediate care doctor can use the vital signs, Glasgow coma scale, and $\mathrm{SaO}_{2}$ as objective means of assessing and monitoring the patient's general condition, enabling treatment to be started at the earliest sign of deterioration. "' A fall in $\mathrm{SaO}_{2}$ may be the first sign, particularly if the cause is airway obstruction or hypoventilation. Monitoring the trend in $\mathrm{SaO}_{2}$ throughout the prehospital phase of care permits observation of the patient's response to treatment, which may also be of value in training and research. The hospital doctor receiving the critically injured casualty can be provided with valuable information from the roadside. Moreover, patients whose condition is deteriorating or who have failed to respond to resuscitation may rapidly be identified by remeasuring the trauma score and $\mathrm{SaO}_{2}$ on arrival in the accident and emergency department.

This study shows that pulse oximetry can be performed during the prehospital phase of a patient's care and that it has considerable benefits in terms of diagnosis and management of the injured patient. The use of pulse oximeters in hospital is increasing, and their cost (currently ranging between $£ 1000$ and $£ 2500$ per unit, depending on the degree of refinement) seems certain to fall as competition among manufacturers increases. Though it would not be feasible for every immediate care doctor and front line ambulance to be equipped with a pulse oximeter, it does seem reason- 
able that second response teams concerned in the management of trapped casualties and mass casualty incidents should carry one. The delivery of trauma care in the United Kingdom is currently under review, and the combination of pulse oximetry and trauma scoring at the roadside and later in the accident and emergency department should allow assessment of the effectiveness of immediate care to be made.

I thank Ohmeda and MAGPAS for their support in this study, Dr N A Silverston and Mr B B Milstein for their help in presenting the results, and Miss Nicola Townley for the photograph.
2 Champion HR, Sacco WJ, Carnazzoj AJ. The trauma score. Cin Care Med 1981;9:672-6

3 Silverston P. The PuLA: a new frontline airway? gournal of the British Asssociation of Immediate Care 1989;12:8-10.

Silverson P. The MAST suit Ambalane fow

+ Silverston P. The MAST sult. Ambulance Fourmal 1980;10:153-60.
5 Zorab JSM. Who needs pulse oximetry? Br Med F 1988;296:658-9.

6 Mihm FG. Halperin BD. Non-invasive detection of profound arterial desatur tion using a pulse oximetry device. Anesthesiologv 1985;62:85-7

7 Yelderman M, New W. Evaluation of pulse oximetry. Anesthesiology 1983;59: $349-52$.

8 Galdun J, Dunmire S, Caplan RM, Heler MB. Accuracy of transcutaneous, transconjunctival and pulse oximetry during air and ground transport with induced hrpoxia and hyperoxia. Ann Emerg Med 1988;17:188.

9 McGuire TJ, Pointer JE. Evaluation of a pulse oximeter in the pre-hospital setting Ann Emerg Med 1988:17:188.

10 Teasedale Gi Jennell B. Assessment of coma and impaired consciousness: a practical sale. Lancet 1974;ii:81.

\title{
HIV viral sequences in seronegative people at risk detected by in situ hybridisation and polymerase chain reaction
}

\author{
M Pezzella, P Rossi, V Lombardi, V Gemelli, R Mariani Costantini, M Mirolo, C Fundaro, \\ $\mathrm{V}$ Moschese, $\mathrm{H}$ Wigzell
}

\section{Abstract}

A study was conducted to assess the occurrence of latent infection with the human immunodeficiency virus (HIV) among seronegative people at high risk of infection. The presence of HIV genomes was analysed by molecular techniques in two seronegative children born to mothers infected with HIV and in three regular sexual partners of seropositive drug addicts. The adults were selected from a seronegative cohort at high risk of infection because of their sexual contacts and the children selected because of impaired growth. HIV retroviral sequences were detected in four of the five subjects directly at the cellular level by in situ hybridisation in peripheral blood mononuclear cells. HIV genomic sequences were confirmed by in vitro amplification of viral DNA with the polymerase chain reaction technique.

The existence of a latent viral infection state in these seronegative subjects indicates the unreliability of standard serological analysis in people who have been in regular contact with infected patients.

\section{Introduction}

After infection with the human immunodeficiency virus (HIV) viral genetic information becomes integrated in the genome of infected cells.' Epidemiological and clinical observations support the hypothesis that the virus may establish a latent infectious state characterised by low viral expression and the absence of a detectable specific antibody response..$^{2}$ Moreover, isolation of the virus by coculture techniques in seronegative subjects at risk $^{+}$suggests that serological analysis is not fully predictive of the infectious state and that infected seronegative people may be a potential source of uncontrolled spread of the virus. Recently high resolution molecular techniques have been described that allow the identification of viral genomes even if only a few copies of integrated or non-integrated viral DNA are present. In situ hybridisation with DNA or RNA (ribonucleic acid) probes gives qualitative information and accurately reflects the distribution of HIV viral genomes at the single cell level. ${ }^{5}$ The polymerase chain reaction technique, a new and sensitive method of gene amplification, allows the selective enrichment of a specific DNA sequence by a factor of $10^{\circ}$ These techniques might be of particular value in detecting HIV viral sequences when numbers of copies of cloning DNA (cDNA) and RNA per cell are very low.

We report using DNA-DNA, DNA-RNA, and RNA-RNA in situ hybridisation and polymerase chain reactions to identify HIV DNA and RNA in peripheral blood mononuclear cells from seronegative subjects at risk of infection-namely, children born to infected mothers and regular sexual partners of people infected with HIV.

\section{Patients and methods}

The study sample consisted of $(a)$ two seronegative women who were regular sexual partners of men infected with HIV (Centers for Disease Control group 3 and 2$) ;(b)$ a seronegative bisexual man who in the past two years had had sexual intercourse with infected partners; and $(c)$ two seronegative children aged 18 and 24 months born to asymptomatic seropositive mothers. The adult sample was collected from among a cohort of partners of people at risk attending the outpatient clinic of the National Centre for Blood Transfusion, Rome. This particular group was selected on the basis of the frequency of unprotected sexual contact with infected drug addicts. The two children were selected from a group of seronegative infants born to infected mothers and who had a history of impaired growth. All subjects were tested for specific serum antibodies to HIV by a commercial enzyme linked immunosorbent assay (ELISA) (ELAVIA; Pasteur) and by western blot analysis (Pasteur). Analysis of free antigen in the serum by an antigen capture assay (Abbott) was also performed. Immunological investigations included total T cell count and CD4 and CD8 phenotyping with commercial monoclonal antibodies in a fluorescence activated cell sorter (Becton Dickinson). All the subjects were enrolled for clinical and laboratory follow up and were monitored for more than one year.

\section{DNA-DNA AND DNA-RNA IN SITU HYBRIDISATION}

Heparinised peripheral blood samples were centrifuged over a Ficoll-Hypaque density gradient (Pharmacia, Sweden). Mononuclear cells collected at the interface were rinsed twice in Roswell Park Memorial Institute 1640 medium (Flow Laboratories) 